\title{
A simple breathing circuit allowing precise control of inspiratory gases for experimental respiratory manipulations
}

Felipe B Tancredi ${ }^{1,23^{*}}$, Isabelle Lajoie ${ }^{1,2,3}$ and Richard D Hoge $\mathrm{e}^{1,2,3}$

\begin{abstract}
Background: Respiratory manipulations modulating blood flow and oxygenation levels have become an important component of modern functional MRI applications. Manipulations often consist of temporarily switching inspired fractions of $\mathrm{CO}_{2}$ and $\mathrm{O}_{2}$; and have typically been performed using simple oxygen masks intended for applications in respiratory therapy. However, precise control of inspired gas composition is difficult using this type of mask due to entrainment of room air and resultant dilution of inspired gases. We aimed at developing a gas delivery apparatus allowing improved control over the fractional concentration of inspired gases, to be used in brain fMRI studies.

Findings: The breathing circuit we have conceived allowed well controlled step changes in $\mathrm{FiO}_{2}$ and $\mathrm{FiCO}_{2}$, at moderate flow rates achievable on standard clinical flow regulators. In a two run test inside the scanner we demonstrate that tightly controlled simple gas switching manipulations can afford good intra-subject reproducibility of induced hyperoxia/hypercapnia responses. Although our approach requires a non-vented mask fitting closely to the subject's face, the circuit ensures a continuous supply of breathable air even if the supply of medical gases is interrupted, and is easily removable in case of an emergency. The apparatus we propose is also compact and MRI compatible, allowing subject placement in confined spaces such as an MRI scanner for brain examinations.
\end{abstract}

Conclusions: We have reported a new approach for the controlled administration of medical gases, and describe an implementation of the breathing circuit that is MRI compatible and uses commercially available parts. The resultant apparatus allows simple, safe and precise manipulations of $\mathrm{FiO}_{2}$ and $\mathrm{FiCO}_{2}$.

Keywords: Respiratory manipulation, Functional MRI, Hypercapnia, Hyperoxia, Breathing circuit

\section{Background}

Respiratory manipulations modulating the fractional concentration of inspired $\mathrm{O}_{2}$ and $\mathrm{CO}_{2}\left(\mathrm{FiO}_{2}\right.$ and $\left.\mathrm{FiCO}_{2}\right)$ to induce hyperoxia/hypercapnia and modulate cerebral blood flow and oxygenation have become an important component of fMRI studies measuring the vascular as well as the metabolic function in the brain [1-7]. Methods using computerized systems to control modulations in inspired concentrations and target specific end-tidal levels have been proven successful in enabling inter-subject reproducibility of hyperoxic/hypercapnic stimuli [8-13] as

\footnotetext{
* Correspondence: felipe.tancredi@umontreal.ca

'Institut de génie biomédical, Département de physiologie, Université de

Montréal, C.P. 6128, Succursale Centre-ville, Montréal, Québec H3C 3J7, Canada

${ }^{2} U$ nité de neuroimagerie fonctionnelle, Centre de recherche de l'institut

universitaire de gériatrie de Montréal, Montreal, QC, Canada

Full list of author information is available at the end of the article
}

well as in providing flexibility in the achievable gas mixtures [14-16] to test different hypotheses. While individualized mixing of gas concentrations to achieve prospective control of end-tidal levels is useful in certain situations, the requirements for many applications are met by delivering predetermined concentrations of inspired gases, so long as end-tidal values are recorded for retrospective normalization of the fMRI signal. The latter, simpler, approach thus continues to be widely adopted in quantitative neuroimaging and other areas. A common practice has been to administer fixed fractional concentrations of $\mathrm{O}_{2}$ and $\mathrm{CO}_{2}$ for inhalation [3,5,6,17], delivering $\mathrm{O}_{2} / \mathrm{CO}_{2}$ enriched mixtures through low-cost nonrebreathing masks commonly used in clinical oxygen therapy, which usually incorporate a reservoir bag to increase dosage efficiency (the bag reservoir stores the gases delivered during expiration to make it available for next inspiration(s), 
making better use of administered gases and alleviating dropouts in $\mathrm{FiO}_{2}$ and $\mathrm{FiCO}_{2}$ during early phases of inspiration) [18]. However, either due to variations in the shape of the subject's face or to incomplete sealing of one-way valves used to release excess gas flow, such oxygen masks are often very leaky [18], hampering precise adjustments in fractional inspired concentrations and thus limiting the reproducibility of the hyperoxic/ hypercapnic stimuli. One way around this limitation would be to ensure a tight seal of the face mask using surgical tape or other means. However, in a closed circuit where the gas supply is directly connected to the mask, this creates a risk of asphyxia in the event that the medical gas supply is interrupted, although this can be mitigated through the incorporation of special safety measures. To improve control over inspired gases we have conceived a breathing circuit whose different compartments are separated by high efficiency one-way valves and whose breathing chamber consists of a non-vented mask providing a tight facial fit, but that can rapidly removed. To ensure the safety of subjects, we have replaced the breathing bag found in oxygen masks by an open reservoir, through which the subject breathes room air whenever the flow of administered gases becomes insufficient. The resulting open breathing circuit (Figure 1) represents an inherently safe design and allows precisely controlled step changes in the fractional concentration of inspired gases, with moderate flow rates.

\section{Findings}

\section{Material and methods}

The breathing circuit we describe below (Figure 2) has been implemented, using commercially available components, to address three design criteria: MRI-compatibility, accommodation within MRI radiofrequency coils enveloping the head for brain exams, and sufficiently low cost to justify disposal after use to avoid transmission of airborne or other pathogens. Parts were acquired from two different vendors: Intersurgical Inc (NY, USA) and Teleflex Medical (NC, USA); which we will be referred to as IS and TM respectively.

The circuit comprises a small non-vented face mask (IS \#7193) and a dual-limb airway that is appended to the mask's frontal opening. An elbow (TM \#1632 or \#1624) and a triple swivel wye-piece (IS \#1929) connect the limbs to the mask. A pair of valves (TM \#1664/5) at the join of the limbs ensure that 1) inspired gases only come from the incoming limb, consisting of a corrugated tube ( 2 TM \#1418 connected by TM \#1960) that is preceded by a connector (TM \#1642); and 2) that expired gases only flow through the outgoing limb, that can be a short corrugated tube (TM \#1410). These two limbs have an open end, communicating with the exterior. While the small outgoing limb serves as an exit for expired gases, the long incoming limb serves as a gas reservoir, like the breathing bag of conventional oxygen masks. However, because of its geometry (we used $\sim 3.5 \mathrm{~m}$ of corrugated tube with $22 \mathrm{~mm}$ internal diameter) and open end, the limb reservoir functions as a sequential container, where administered gases are stacked as they arrive at the circuit and can be expected to mix less than in a bag type of reservoir. This way, during transitions between gas mixtures of different concentrations, the new mixture becomes readily available for inspiration, allowing sharp transitions in fractional concentration of inspired gases. Whereas the same effect could be achieved without this long limb reservoir, that would require high flow dosages to meet peak inspiratory rates.

The circuit was conceived to allow better isolation between circulating gases and exterior, while ensuring a constant supply of breathable gas even in the event of an interruption in the gas administration. The anesthetic mask we have adopted offers a tight facial fit, drastically reducing entrainment of room air and resultant dilution of inspired/expired gases (as can occur with Hudsonstyle oxygen masks) but can be very easily removed in case of danger or discomfort. The mask does not need to be sealed using adhesive tape [8] nor held to the head using restrictive harnesses that can be difficult to remove in an emergency [19] (we have adopted the TM \#15920). More importantly though, as mentioned above, the gas reservoir of our circuit has an open end which protects the subject against disruptions in the gas supply. Whenever the gas administration is insufficient to meet the subject's ventilation the reservoir is replenished with room air for inspiration, i.e. there is no special requirements to ensure a constant supply of breathable air.

The proposed circuit is also equipped with a sampling port for the monitoring of $\mathrm{O}_{2} / \mathrm{CO}_{2}$ and pressure wave inside the mask chamber, avoiding the use a nasal cannula and potential discomfort associated with this. The sampling port is positioned at the elbow that connects the mask to the circuit's limbs and can be of two types, barbed (elbow TM \#1632) or luer lock (elbow TM \#1624), depending on the tubing used for the sampling line.

As a proof of concept of the improvement in respiratory manipulations attained with the new circuit we have conducted two tests using both the new circuit and a nonvented Hudson oxygen mask (\#1060) in a young healthy subject (female: $32 \mathrm{y}, 1.65 \mathrm{~m}, 60 \mathrm{~kg}$ ). In Test 1 the subject was given $100 \% \mathrm{O}_{2}$ or $50 \% \mathrm{O}_{2}$ balanced with air in two different instances lasting 3 minutes each and 3 minutes apart. Medical air was administered otherwise. Flow rates were $15 \mathrm{~L} / \mathrm{min}$ during the whole manipulation. Test 2 followed the same design as Test 1 but with different flow rates. Upon transitions in administered concentrations, flow rates were increased to $30 \mathrm{~L} / \mathrm{min}$ for $1 \mathrm{~m}: 30 \mathrm{~s}$. 


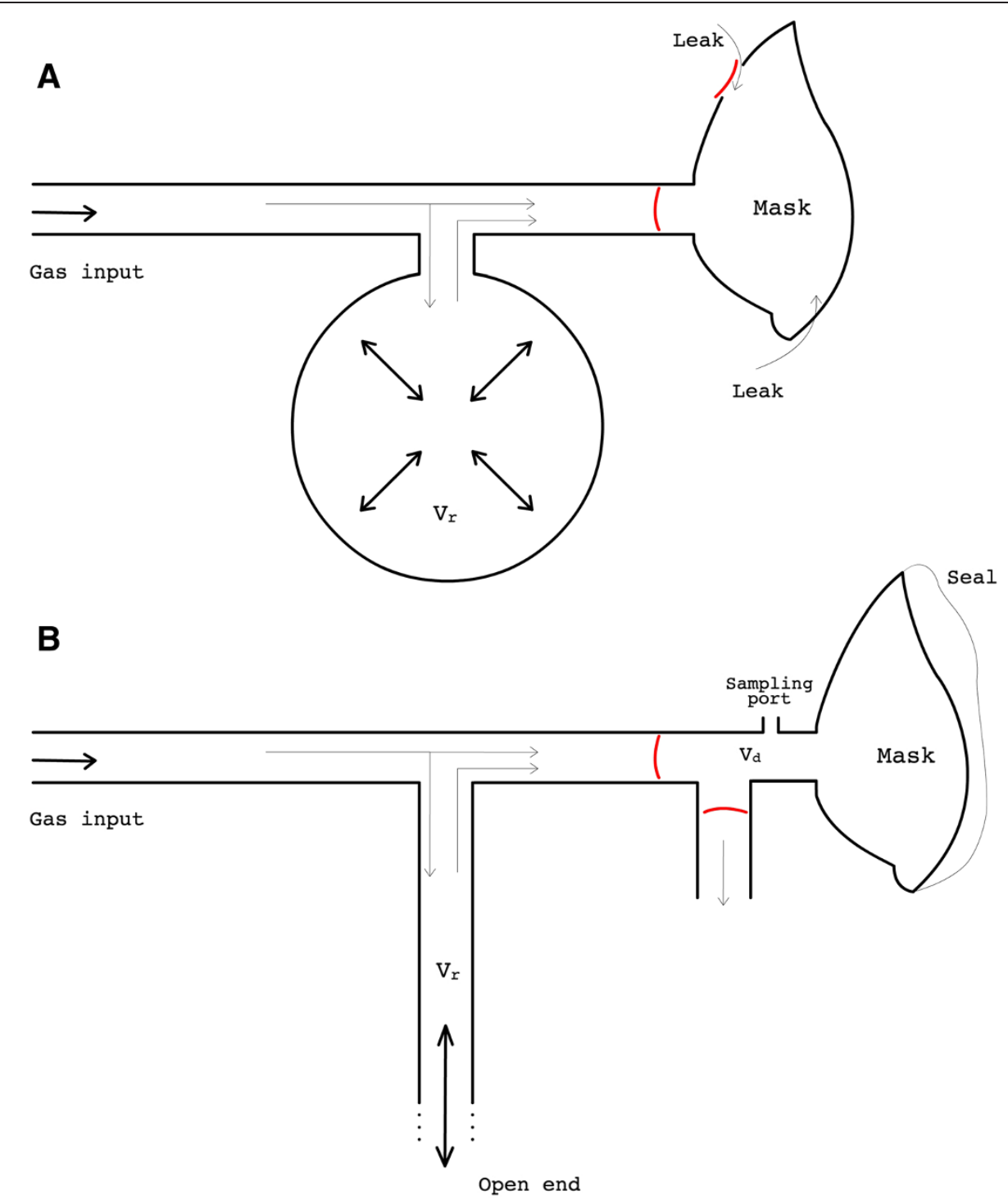

Figure 1 Schematic comparison between the new breathing circuit and a non-rebreathing oxygen mask. A typical oxygen mask/circuit is shown on the top (A). The input gas may go straight to the mask or fill the breathing bag when delivery rate exceeds uptake rate (e.g. during the expiratory phase of the breathing cycle). Whenever uptake exceeds delivery rate the bag empties to provide supplementary gas for inspiration. Expired gases leave the mask through vents controlled by valves. Gases inside the mask are often contaminated by air that leak in through the edges of the mask and/or through the vents when valves are malfunctioning. This compromises control over the inspiratory gases as well as the accurate monitoring of end-tidal values. To avoid the latter concerns the new breathing circuit we have developed (B) uses a non-vented mask providing a sealed fit to the subject's face. But also, the breathing bag is replaced by a long limb reservoir with an open end, which makes the circuit completely safe as the person can normally breathe room air if no gas is supplied. Besides, the geometry of such reservoir permits faster transitions in $\mathrm{FiO}_{2}$ and $\mathrm{FiCO}_{2}$. Expired gases are exhausted through a second limb, controlled by an efficient unidirectional valve. Lastly, a sampling port is installed adjoined to the mask to help the respiratory monitoring. $\mathrm{Vr}=$ volume of the reservoir; $\mathrm{Vd}=$ dead space added by appending the dual limb system to the mask.

The new circuit has been deployed in multi-subject fMRI experiments conducted by our group, in which $\mathrm{FiO}_{2}$ and $\mathrm{FiCO}_{2}$ were modulated administering $100 \% \mathrm{O}_{2}$ and $5 \%$ $\mathrm{CO}_{2}$ according to the schedule proposed by Bulte et al. [7]. The circuit has been used in over 20 MRI sessions, with very consistent results. We will show here data obtained from single representative participant who underwent two different runs of the above protocol.
Respiratory gases were monitored using Biopac MP150 (Biopac Systems Inc., CA, USA). Fractional concentrations of $\mathrm{O}_{2}$ and $\mathrm{CO}_{2}$ were analyzed and recorded at a $50 \mathrm{~ms}$ sampling rate. The gas sampling line consisted of a long segment of rigid tubing pertaining to the monitoring equipment (AFT31-XL) preceded by a disposable bacterial filter (\#2200/01, Air Safety Medical Ltd,) and a short segment of oxygen tubing (\#2001, Salter Labs, CA, USA) to 


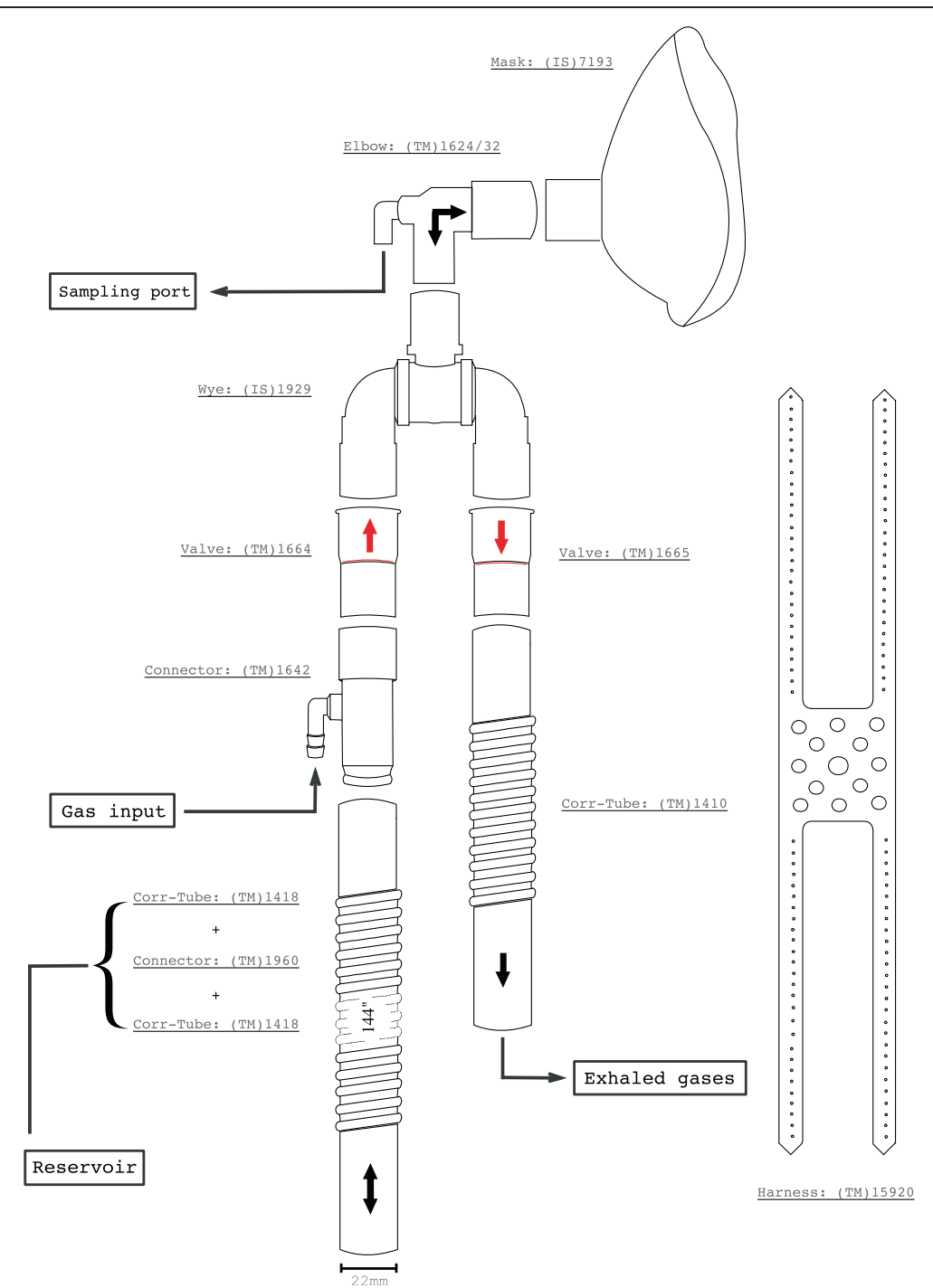

Figure 2 Schematic of the circuit assembly. Gas mixtures are supplied through the barbed inlet of the connector TM \#1642. The elbow can be of two different types, with a luer-lock or barbed sampling port (TM \#1624 or \#1632 respectively). The limb reservoir is formed by two corrugated tubings (TM \#1418) coupled by a connector (TM \#1960), which is not represented here. The mask (IS \#7193) comes with a support (not represented) allowing to attach the harness (TM\# 15920).

connect the filter to the sampling port of our breathing circuit (built using a TM \#1632 elbow). We noted that, using the current setup, accurate measures of fractional concentration were difficult to obtain when breathing rates exceeded 10 breaths per minute. We believe this effect is mainly associated with the limited rise/fall time of our gas analyzers and mixing of gases inside the sampling line; but we have also observed that the bacterial filter placed in the sampling line contributes to the problem, although we are not certain through which exact mechanisms. In future experiments we intend to 1) replace the long segment of the sampling line by a narrow-bore plastic tubing (then, to overcome the resistance it adds to the line, attach an external sampling pump to Biopac) and 2) test other solutions to filter the sampling gases. In the present study, however, to obtain crisp respiratory signal traces we have instructed subjects to limit their respiratory rate to 10 breaths per minute. If the subject breathes at a pace lower than usual there may be less contamination of inspired gases in breathing circuit, which will result in better performance controlling for inspired doses (even if the decrease in respiratory rate is just minor such as in the particular case of the herein experiments). However, the same is true for the oxygen mask, i.e. a reduced respiratory rate also lessens contamination of inspired gases and improves the mask's performance. Therefore we considered that the special requirement to cap respiratory rate should not invalidate the comparison between performances of the two apparatuses. On the contrary, we considered that it would help better illustrating the 
differences one should observe if using a robust sampling method.

The pressure wave at the end of the sampling line was monitored using an in-house transducer that connected to one of Biopac's analog channels. This signal helped identifying the expired (i.e. end-tidal) points in the post processing of $\mathrm{O}_{2}$ and $\mathrm{CO}_{2}$ respiratory traces.

Fractional concentrations were multiplied by $760 \mathrm{mmHg}$ to be converted in approximate partial pressure values. Baseline levels and changes in end-tidal partial pressures were quantified using the approach described in ref [17]. The end-tidal sampled points were fit to a linear model consisting of a third degree polynomial term plus the $\mathrm{CO}_{2} / \mathrm{O}_{2}$ administration periods as the response regressors, which were shaped using bi-exponential functions to improve the contour of transitions. Baseline levels were obtained from the regressors representing the offset term of the model; respiratory responses represented the effect size of fitted response regressors.

\section{Results}

In Figure 3 we show results of Tests 1 and 2; tests with the oxygen mask are shown on the left column whereas test with the new circuit on the right.

From the plots on the left column we note how different the composition of actual inspired gases $\left(\mathrm{FiO}_{2}\right)$ and the composition of administered gases (FA) can be when the oxygen mask is used. Furthermore, in the second row we have a clear example of how dependent the composition dosage - i.e. $\mathrm{FiO}_{2}-$ can be on the flow dosage - i.e. flow rate (FR). The administration of $100 \% \mathrm{O}_{2}$ at different FR's induces different $\mathrm{FiO}_{2}$ inputs (as well as end-tidal responses). This is related to the contamination of administered gases by room air that leaks in to the mask: for lower FR's the pressure inside the mask is lower, which worsens the leak and reduces $\mathrm{FiO}_{2}$. Conversely, when fixed FR's are used (which is often the case) the degree of leak will vary according to the fit of the oxygen mask to the subjects' face. This compromises inter- but as well intra-subject reproducibility of the $\mathrm{FiO}_{2}$ manipulations.

In the tests with the new circuit (right column) there was a very close correspondence between $\mathrm{FA}$ and $\mathrm{FiO}_{2}$. In the test where FR was kept constant throughout the manipulation (first row) we note that the transitions in $\mathrm{FiO}_{2}$ were not as sharp as the switching of gases. This results from the small contamination of new input gases with gases that remained in the limb reservoir from the preceding input phase. In the test where FR is increased from $15 \mathrm{~L} / \mathrm{min}$ to $30 \mathrm{~L} / \mathrm{min}$ upon the switching of gases (second row) the replacement of gases from the preceding input by the gases constituting the new FA input is faster, which results in $\mathrm{FiO}_{2}$ transitions that are sharper. Such maneuver can be used in other experiments as a means to achieve squared step changes in inspired doses.
However, in this particular manipulation, the average FR was increased by $100 \%$ of the regular flow rate - the limb reservoir being flooded with approximately $22 \mathrm{~L}$ of excess gases during each of the $1 \mathrm{~m}: 30 \mathrm{~s}$ transitional periods. To keep the average FR close to normal levels and minimize gas usage, the flush procedure should be as fast as possible. Ideally one would deliver a short bolus of the new FA input with the approximate volume of the limb reservoir. Nevertheless, the flush does not need to be well controlled and in fact any increase in flow rate has the effect of accelerating the transitions in inspired doses. For instance, if flow rates must be limited to a maximum of $20 \mathrm{~L} / \mathrm{min}$, keeping FR at this level for 2030 seconds will greatly improve transitions in the inspired doses. Yet, in the many cases where traceability of flow rates is not a concern, the simplest approach to flush the limb reservoir would consist of opening the regulator's valve widely just before setting the flow rate to normal, traceable, levels.

In Figure 4 we show results from manipulations using the circuit in a fMRI experiment for the non-invasive measurement of $\mathrm{CMRO}_{2}$ [5]. The 18-minute respiratory schedule proposed by Bulte et al. [7], which includes interleaved stimuli of hyperoxia and hypercapnia, was repeated twice. To accelerate transitions in inspired doses, we have momentarily increased FR from $15 \mathrm{~L} / \mathrm{min}$ to $60 \mathrm{~L} / \mathrm{min}$ during 3 seconds upon each FA transition, flooding the limb reservoir with $3 \mathrm{~L}$ boluses before resumption of the regular flow rate. The change in gas flow during this flushing procedure is greatly attenuated when gases reach the mask (due to dispersion in the long tubes), and is only marginally perceptible to the subject.

The dosage efficiency was again high, as evidenced by the tight correspondence between the fractional composition of the administered mixtures and the fractional composition of the actual inspired gases. In the $\mathrm{O}_{2}$ monitoring signal we see the same squared $\mathrm{FiO}_{2}$ contours as observed in Figure 3 when the $100 \% \mathrm{O}_{2}$ is administered; all of which have the same height as the FA of $\mathrm{O}_{2}$, i.e. $760 \mathrm{mmHg}$. In the $\mathrm{CO}_{2}$ monitoring signal, we find blank, rectangular areas under the $\mathrm{CO}_{2}$ trace when the $5 \% \mathrm{CO}_{2}$ mixture is administered. The height of these areas correspond well to the FA of $\mathrm{CO}_{2}$. Using moderate rates and a simple flushing procedure, our respiratory circuit allowed sharp and precisely controlled step changes in both $\mathrm{FiO}_{2}$ and $\mathrm{FiCO}_{2}$.

Our test results also demonstrate that end-tidal responses to controlled step changes in inspired concentrations can be quite reproducible. In Figure 4 it can be seen that modulations in end-tidal gas levels are very similar both in terms of their qualitative shape and their amplitude. In each plot, the leftmost value represents the baseline end-tidal level of the respective gas, whereas the 4 other values represent the responses associated to 


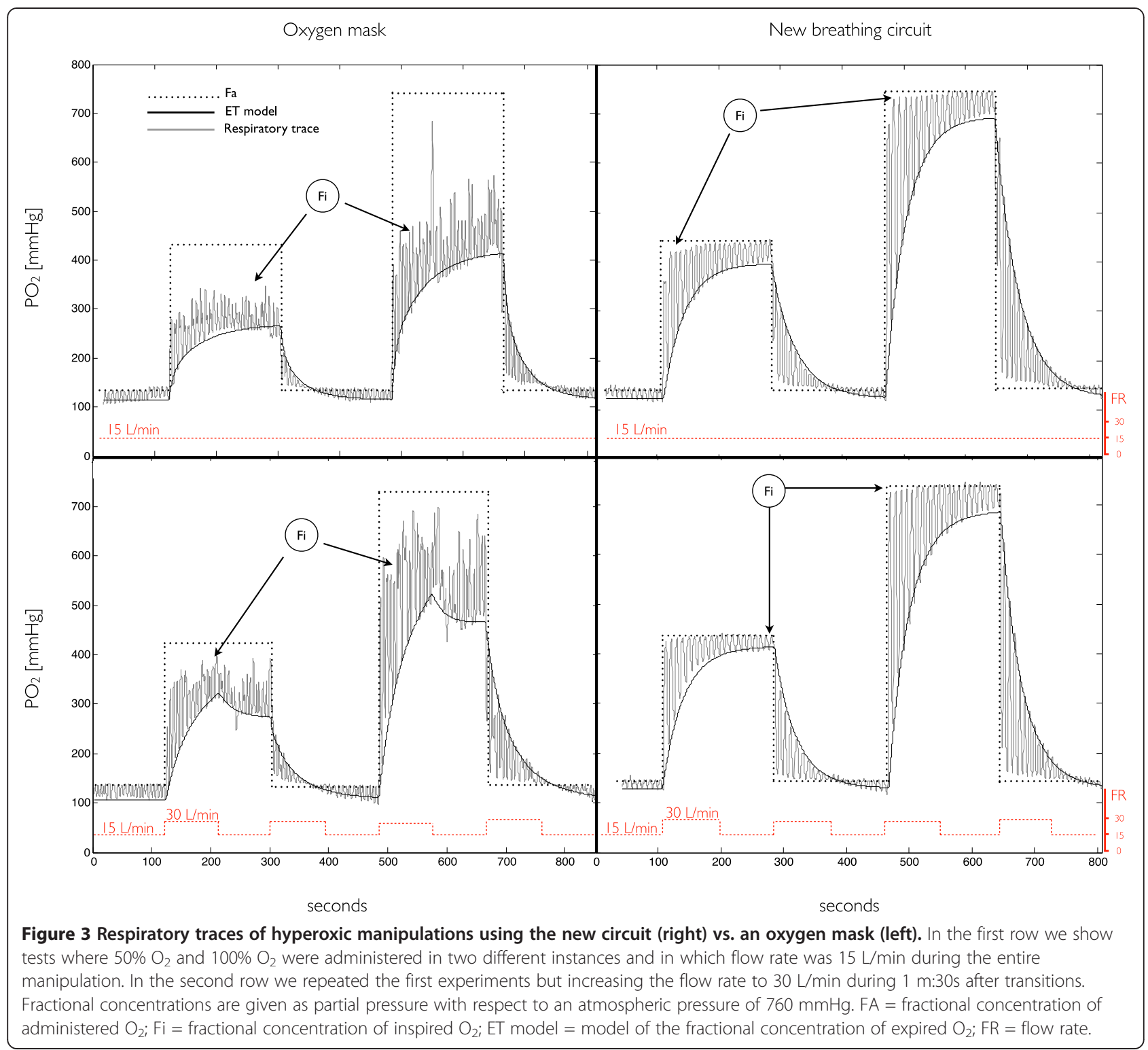

the switching of administered/inspired compositions. Modeled increases in $\mathrm{P}_{\mathrm{ET}} \mathrm{O}_{2}$ and $\mathrm{P}_{\mathrm{ET}} \mathrm{CO}_{2}$ were both consistent: $\mathrm{P}_{\mathrm{ET}} \mathrm{O}_{2}$ increases varied by less than $10 \%$ relative to the baseline levels whereas $\mathrm{P}_{\mathrm{ET}} \mathrm{CO}_{2}$ increases varied by less than $2 \%$. Since the magnitude of elicited responses to a given challenge depend on the physiological status of the individual, we note that it might not be possible to attain the same degree of intra-subject reproducibility when subjects are scanned in multiple days.

A careful inspection of the $\mathrm{O}_{2}$ monitoring trace reveals a small negative $\mathrm{FiO}_{2}$ input during the administration of $\mathrm{CO}_{2}$. This results from the slightly lower oxygen content in the administered $\mathrm{CO}_{2}$ mixture which is composed of $5 \% \mathrm{CO}_{2}$ and $95 \%$ of air. And we also note that in this scheme 1) hyperoxic stimuli may not be isocapnic, i.e. when breathing pure $\mathrm{O}_{2}$ the subject's $\mathrm{ETCO}_{2}$ can be markedly depressed; 2) nor the hypercapnic stimuli perfectly isooxic as $\mathrm{ETO}_{2}$ also changes when the $\mathrm{CO}_{2}$ mixture is administered (although this may be less of a concern given the small, $<5 \%$, relative changes in $\mathrm{O}_{2}$ levels and its respective impact on the MRI signal).

\section{Conclusion}

We have developed a simple breathing circuit allowing well controlled step changes in the fractional concentration of inspired gases, to be used in applications such as in MRI studies measuring the cerebrovascular responses to respiratory gases. In addition, the circuit ensures uninterrupted availability of gas and operates at moderate flow rates achievable with standard clinical flow regulators. 


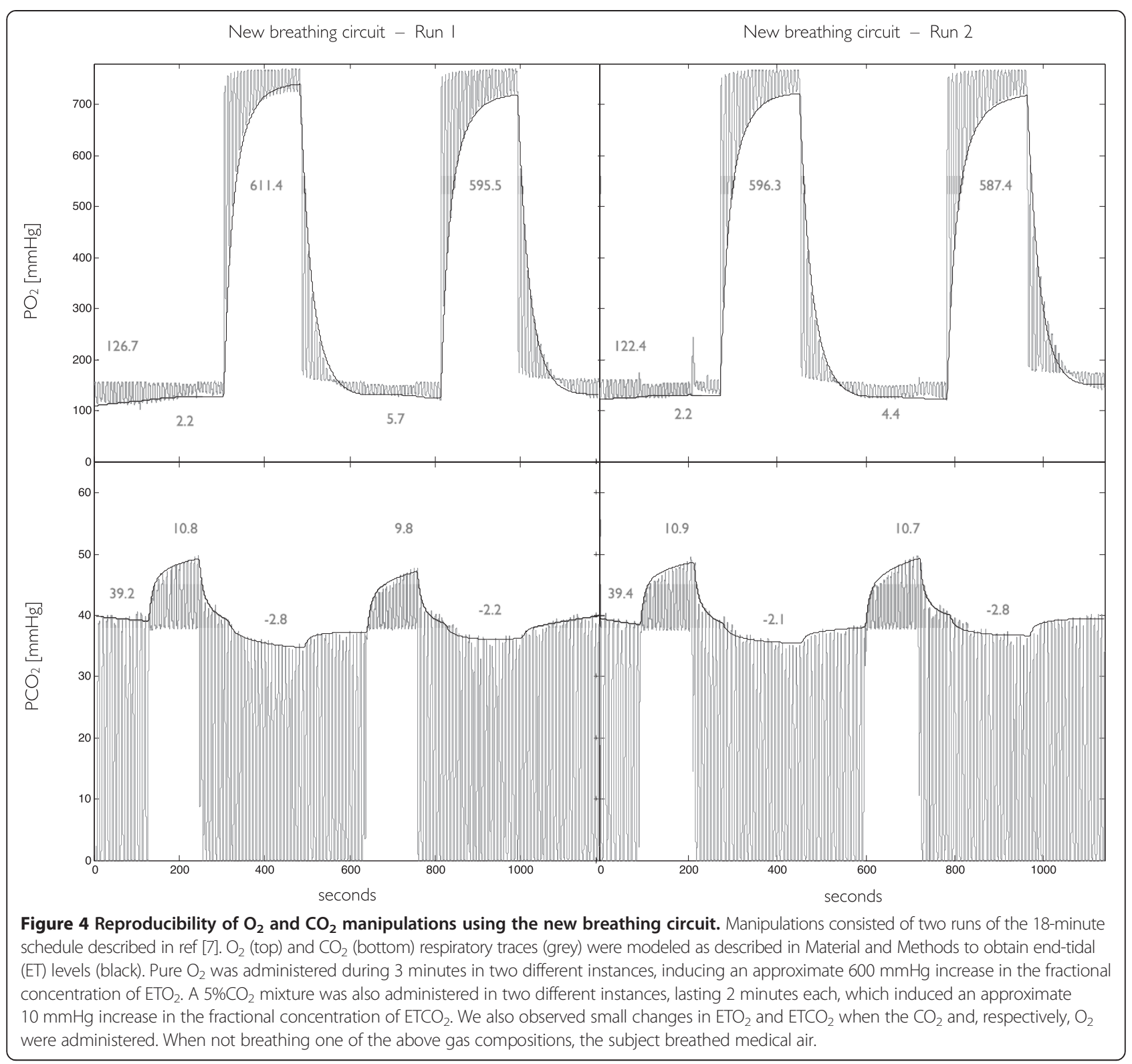

The type of mask and unidirectional valves we have employed in our circuit, which prevent dilution of administered gases by room air (as can occur when using standard clinical oxygen masks), are the features permitting the control over fractional inspired concentrations. However, the key component of our circuit is the limb reservoir. While optimizing gas consumption, it permits sharper transitions in the fractional inspired concentrations and, more importantly, endows the circuit with a fail-safe mechanism. It should be emphasized that for the proper operation of the circuit and its safety features, the limb reservoir must be kept unobstructed at all times. To ensure total protection of the subject additional safety measures should be included, such as the ongoing and careful monitoring of both inspired and expired respiratory gases, as well as arterial $\mathrm{O}_{2}$ saturation.

Although the proposed circuit could also be used in applications entailing frequent adjustments in inspired fractions (e.g. for feed-back control of end-tidal levels) that would require high flow rates to permanently flush the limb reservoir with the right gas composition for inhalation. To control of end-tidal levels at low flow rates alternative methods are warranted [12]. Using the sequential gas delivery method introduced by Banzet et al. [11] - which allows controlling for the effective volume of inspired mixtures - combined with a physiological model of the dependence of end-tidal responses on inspired fractions it is possible to prospectively target end-tidal levels with minimal gas usage (a feed-forward modeling method 
as opposed to feed-back control). This approach requires a computer controlled mixing system in addition to a specially designed breathing circuit, which is normally taped on the subjects' face and forms a closed loop (in case of discomfort the subject can remove a safe plug positioned near the nose).

The prototype we have devised using commercially available parts has a dead space that prevented us from having perfectly squared step changes in inspired concentrations. As can be noticed in most transitions, inspired levels in the first breath right after the switch of gases do not attain the administered concentrations. Ideally the circuit should be fabricated using a non-vented and tightly-sealing mask with minimal-size chamber and a gas input as close as possible to the subjects nose/ mouth to minimize the dead space. Such a circuit, like the one presented here, should include sampling ports, to avoid the use of nasal cannulas and the potential discomfort associated with these, and be disposable.

In summary, the breathing circuit we have presented 1) allows control over fractional inspired concentrations, 2) optimizes gas consumption in block design manipulations, 3) is simple, 4) safe and 5) comfortable.

\section{Competing interests}

The authors declare that they have no competing interests.

\section{Authors' contributions}

FBT contributed to the conception and design of the experiments, data collection and analysis, interpretation of the results, and took the lead role in preparation of the manuscript. IL contributed to the data collection, interpretation of results and preparation of the manuscript. $\mathrm{RDH}$ contributed to the design of the experiments, interpretation of the results, and preparation of the manuscript. All authors read and approved the final manuscript.

\section{Acknowledgements}

We would like to thank Anne-Marie Bédard for help with technical and administrative support; Marius Tuznik with data acquisition; and Francine Beausejour and Louise Gervais (from the Dept. of Respiratory Therapy - Institut universitaire de gériatrie de Montréal) for their expert opinion. This work was supported by Consortium québéquois sur la découverte du médicament, Canadian Foundation for Innovation (Leaders Opportunity Fund 17380), Canadian Institutes of Health Research (MOP-273379), Natural Sciences and Engineering Council of Canada (355583-2010) and MITACS (IT02305 scholarship received by F.B.T.).

\footnotetext{
Author details

${ }^{1}$ Institut de génie biomédical, Département de physiologie, Université de Montréal, C.P. 6128, Succursale Centre-ville, Montréal, Québec H3C 3J7, Canada. ¿Unité de neuroimagerie fonctionnelle, Centre de recherche de l'institut universitaire de gériatrie de Montréal, Montreal, QC, Canada. ${ }^{3}$ Centre de recherche de l'institut universitaire de gériatrie de Montréal, 4545, Queen Mary, Montreal, QC H3W 1W5, Canada.
}

Received: 22 October 2013 Accepted: 9 April 2014

Published: 12 April 2014

\section{References}

1. Mandell DM, Han JS, Poublanc J, Crawley AP, Stainsby JA, Fisher JA, Mikulis DJ: Mapping cerebrovascular reactivity using blood oxygen level-dependent MRI in patients with arterial steno-occlusive disease: comparison with arterial spin labeling MRI. Stroke 2008, 39:2021-2028.
2. Gauthier CJ, Madjar C, Desjardins-Crépeau L, Bellec P, Bherer L, Hoge RD: Age dependence of hemodynamic response characteristics in human functional magnetic resonance imaging. Neurobiol Aging 2013, 34:1469-1485.

3. Ances BM, Liang CL, Leontiev O, Perthen JE, Fleisher AS, Lansing AE, Buxton RB: Effects of aging on cerebral blood flow, oxygen metabolism, and blood oxygenation level dependent responses to visual stimulation. Hum Brain Mapp 2009, 30:1120-1132.

4. Spano VR, Mandell DM, Poublanc J, Sam K, Battisti-Charbonney A, Pucci O, Han JS, Crawley AP, Fisher JA, Mikulis DJ: CO2 blood oxygen level-dependent MR mapping of cerebrovascular reserve in a clinical population: safety, tolerability, and technical feasibility. Radiology 2013, 266:592-598.

5. Gauthier CJ, Hoge RD: Magnetic resonance imaging of resting OEF and $\mathrm{CMRO}(2)$ using a generalized calibration model for hypercapnia and hyperoxia. Neurolmage 2012, 60:1212-1225.

6. Yezhuvath US, Lewis-Amezcua K, Varghese R, Xiao G, Lu H: On the assessment of cerebrovascular reactivity using hypercapnia BOLD MRI. NMR Biomed 2009, 22:779-786.

7. Bulte DP, Kelly M, Germuska M, Xie J, Chappell MA, Okell TW, Bright MG, Jezzard P: Quantitative measurement of cerebral physiology using respiratory-calibrated MRI. Neurolmage 2012, 60:582-591.

8. Mark C, Slessarev M, Ito S, Han J, Fisher J, Pike G: Precise control of end-tidal carbon dioxide and oxygen improves BOLD and ASL cerebrovascular reactivity measures. Magn Reson Med 2010, 64:749-756.

9. Winter JD, Fierstra J, Dorner S, Fisher JA, St Lawrence KS, Kassner A: Feasibility and precision of cerebral blood flow and cerebrovascular reactivity MRI measurements using a computer-controlled gas delivery system in an anesthetised juvenile animal model. J Magn Reson Imaging 2010, 32:1068-1075.

10. Wise RG, Pattinson KTS, Bulte DP, Chiarelli PA, Mayhew SD, Balanos GM, Connor O, Connor DF, Pragnell TR, Robbins PA, Tracey I, Jezzard P: Dynamic forcing of end-tidal carbon dioxide and oxygen applied to functional magnetic resonance imaging. J Cereb Blood Flow Metab 2007, 27:1521-1532.

11. Banzett RB, Garcia RT, Moosavi SH: Simple contrivance "clamps" end-tidal $\mathrm{PCO}(2)$ and $\mathrm{PO}(2)$ despite rapid changes in ventilation. J Appl Physiol 2000, 88:1597-1600.

12. Slessarev M, Han JS, Mardimae A, Prisman E, Preiss D, Volgyesi G, Ansel C, Duffin J, Fisher JA: Prospective targeting and control of end-tidal CO2 and $\mathrm{O} 2$ concentrations. J Physiol 2007, 581:1207-1219.

13. Robbins PA, Swanson GD, Howson MG: A prediction-correction scheme for forcing alveolar gases along certain time courses. J Appl Physiol 1982, 52:1353-1357.

14. Mutch WAC, Mandell DM, Fisher JA, Mikulis DJ, Crawley AP, Pucci O, Duffin J: Approaches to brain stress testing: BOLD magnetic resonance imaging with computer-controlled delivery of carbon dioxide. PLoS One 2012, 7:e47443.

15. Blockley NP, Driver ID, Francis ST, Fisher JA, Gowland PA: An improved method for acquiring cerebrovascular reactivity maps. Magn Reson Med 2011, 65:1278-1286.

16. Driver I, Blockley N, Fisher J, Francis S, Gowland P: The change in cerebrovascular reactivity between $3 \mathrm{~T}$ and $7 \mathrm{~T}$ measured using graded hypercapnia. Neurolmage 2010, 51:274-279.

17. Tancredi FB, Hoge RD: Comparison of cerebral vascular reactivity measures obtained using breath-holding and $\mathrm{CO} 2$ inhalation. J Cereb Blood Flow Metab 2013, 33:1066-1074.

18. Boumphrey SM, Morris EAJ, Kinsella SM: $100 \%$ inspired oxygen from a hudson mask-a realistic goal? Resuscitation 2003, 57:69-72.

19. Bulte DP, Drescher K, Jezzard P: Comparison of hypercapnia-based calibration techniques for measurement of cerebral oxygen metabolism with MRI. Magn Reson Med 2009, 61:391-398.

doi:10.1186/1756-0500-7-235

Cite this article as: Tancredi et al:: A simple breathing circuit allowing precise control of inspiratory gases for experimental respiratory manipulations. BMC Research Notes 2014 7:235. 